\title{
Tórax inestable en paciente pediátrico. Reporte de un caso
} Flail chest in pediatric patient. Case report

\author{
Dra. Melisa Gamerman ${ }^{a}$, Dr. Enrique Romero Manteola ${ }^{a}$, Dra. Sofía Contreras ${ }^{a}$, Dra. Celeste Patiñ ${ }^{a}$, \\ Dr. Germán Bonetto ${ }^{a}$ Dra. Agustina Salvadores ${ }^{a}$
}

\begin{abstract}
RESUMEN
El tórax inestable se define por fractura de 3 o más costillas adyacentes, en 2 o más sitios, con movimiento paradojal del tórax afectado. Se presenta en el 2-4\% de los traumas de tórax contusos, con una mortalidad del 10-15\%.

El tratamiento se realiza de forma conservadora, mediante analgesia y el uso de ventilación con presión positiva o, quirúrgicamente, mediante osteosíntesis de las fracturas costales.

Se presenta el caso de una niña de 9 años con diagnóstico de traumatismo cerrado de tórax y tóraxinestable. En la tomografía computada, se evidenciaron fracturas costales de $2^{\text {da }}$ a $7^{\text {ma }}$ izquierdas, arcos medios y anteriores. Por inadecuado manejo del dolor, se colocó asistencia ventilatoria mecánica. A las 72 horas, se decidióla resolución quirúrgica mediante osteosíntesis de las costillas $3^{a}-7^{a}$. La paciente evolucionó favorablemente. El manejo quirúrgico permitió reducir los días de ventilación mecánica y de internación.
\end{abstract}

Palabras clave: tórax paradójico, traumatismos torácicos, pediatría.

\begin{abstract}
Flail chest is defined by fracture of 3 or more adjacent ribs, at 2 or more sites, with paradoxical movement of the affected chest. It occurs in 2 to $4 \%$ of blunt chest trauma, with a mortality of 10 to $15 \%$.

Treatment is conservative, using analgesia and positive pressure ventilation, or surgical osteosynthesis of rib fractures.

We present the case of a 9-year-old girl with blunt chest trauma and flail chest. Computed tomography showed rib fractures from $2^{\text {nd }}$ to $7^{\text {th }}$ left ribs, in middle and anterior arches. Due to inadequate pain management, it was placed in mechanical ventilation. After 72 hours the surgical resolution was decided, osteosynthesis of the $3^{\text {rd }}$ to $7^{\text {th }}$ ribs was performed. The patient evolved favorably. The surgical management allowed reducing days of mechanical ventilation and days of hospitalization.

Key words: flail chest, thoracic injuries, pediatrics.
\end{abstract}

http: / / dx.doi.org/10.5546/aap.2020.e57

a. Sanatorio Allende, Córdoba, Argentina.

Correspondencia:

Dra. Melisa Gamerman: melisagamerman@hotmail.com

Financiamiento: Ninguno.

Conflicto de intereses: Ninguno que declarar.

Recibido: 24-1-2019

Aceptado: 18-8-2019
Cómo citar: Gamerman M, Romero Manteola E, Contreras S, Patiño C, et al. Tórax inestable en paciente pediátrico. Reporte de un caso. Arch Argent Pediatr 2020;118(1):e57-e60.

\section{INTRODUCCIÓN}

El tórax inestable (TI) se define por la fractura de 3 o más costillas, en 2 o más sitios, lo que da resultado a un movimiento paradojal del tórax afectado. Es una complicación potencialmente mortal del traumatismo torácico grave y ocurre en hasta el $15 \%$ de las lesiones de la pared torácica, con una mortalidad del $10 \%$ al $15 \% .^{1,2}$

Es una entidad de baja incidencia en pediatría, y se presenta en el 2-4\% de los traumas de tórax contusos. La menor incidencia en comparación con la de la población adulta se debe a la mayor distensibilidad torácica en la edad pediátrica. ${ }^{3}$

Las contusiones pulmonares se asocian entre el $30 \%$ y el $75 \%$ de los casos, lo que aumenta la mortalidad más del $40 \%{ }^{4}$ Las lesiones complejas de la pared torácica pueden afectar la función pulmonar por diversos mecanismos. El dolor lleva a la hipoventilación y a la generación de atelectasias, y compromete también la capacidad para toser y movilizar secreciones. La presencia de hemotórax, neumotórax o hemoneumotórax predispone también el desarrollo de falla respiratoria aguda. ${ }^{3}$

El TI fue descrito por primera vez en el año 1926 por Jones y Richardson. En ese entonces, el concepto de TI se limitaba a una inestabilidad mecánica torácica, y el tratamiento se focalizaba en la inmovilización del segmento afectado. En los últimos 20 años, el tratamiento del TI se focalizó en un manejo conservador. Se centró en mantener una adecuada ventilación e higiene pulmonar, y en el control del dolor por medio de analgesia regional con catéter epidural, técnica con mayores beneficios. ${ }^{5} \mathrm{El}$ último avance en el manejo del TI es la estabilización quirúrgica de las fracturas costales. Esta técnica, aplicada de forma temprana, ha demostrado buenos resultados, con reducción de los días de asistencia ventilatoria mecánica (AVM), complicaciones asociadas y 
estancia en unidades de cuidados intensivos. ${ }^{1}$ Es escasa la bibliografía sobre el manejo de esta patología en los niños.

\section{CASO CLÍNICO}

Se presenta el caso de una paciente de 9 años de sexo femenino, sin antecedentes patológicos de relevancia. La niña fue derivada a nuestra Institución por un traumatismo cerrado de tórax como consecuencia de la caída desde un caballo y su posterior arrastre durante una distancia no determinada.

Ingresó respirando espontáneamente, con soporte de oxígeno por máscara de no reinhalación, saturando al $100 \%$. Tenía múltiples excoriaciones en todo el cuerpo. Se observó deformidad y asimetría torácica izquierda, con respiración paradojal homolateral. Presentaba los siguientes signos vitales al ingresar: tensión arterial de 119/65 mmHg, 25 respiraciones por minuto, frecuencia cardíaca de 117 latidos por minuto (lpm). Escala de coma de Glasgow 15 y un índice de trauma pediátrico de 7 . La paciente se encontraba hemodinámicamente estable y sin signos de insuficiencia ventilatoria.

Por mal manejo del dolor a pesar de haber recibido antiinflamatorios no esteroideos (AINES) y opioides en dosis adecuadas, se decidió colocarle AVM de forma electiva. Se realizó una radiografía de tórax, en la que

FIGURA 1. Radiografía de tórax. Fracturas costales

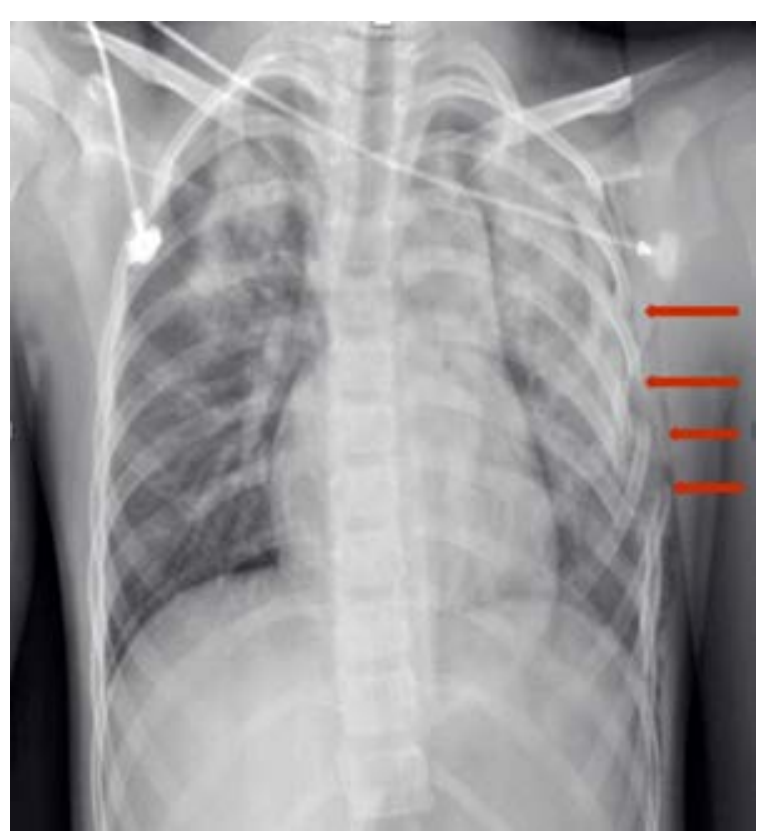

se observaron múltiples trazos de fracturas costales izquierdos, radioopacidad en ambos campos pulmonares, radiolucidez izquierda compatible con neumotórax leve (Figura 1). La tomografía computada (TC) de tórax mostró fracturas costales izquierdas desde la $2^{\mathrm{a}}$ a la $7^{\mathrm{a}} \mathrm{y}$, desde la $4^{\mathrm{a}}$ a la $7^{\mathrm{a}}$, se constataban fracturas en los arcos anteriores y medios, que determinaban el hundimiento y la deformidad, hemoneumotórax y signos de contusión pulmonar (CP) bilateral (Figura 2). La TC de abdomen y de pelvis con contraste, sin evidencia de lesiones. Se colocó un tubo de drenaje pleural. La niña permaneció estable durante 72 horas con relación entre la presión arterial de oxígeno $\left(\mathrm{PaO}_{2}\right.$ y la fracción inspirada de oxígeno $\left(\mathrm{FIO}_{2}\right)$-PaFI- superiores a 300, con buen manejo del dolor y sincronía paciente-ventilador. Se realizó una interconsulta con el Servicio de Cirugía Torácica y se decidió la conducta quirúrgica.

Antes del procedimiento quirúrgico, se realizó una broncoscopía rígida, que descartó la lesión de la vía aérea. Por toracotomía posterolateral izquierda, se realizó osteosíntesis de las costillas 3-7 con clips costales de titanio (clips de Judet). No se realizó osteosíntesis de la $2^{\mathrm{a}}$ costilla por presentar una fractura estable no desplazada. Se verificó la estabilidad costal manualmente (Figura 3 y 4 ).

A las 24 horas posquirúrgicas, se extubó de manera programada; se colocó en ventilación no invasiva (VNI) electiva y se medicó con dexmedetomidina, AINES Y opioides para lograr la adaptación a la VNI y la analgesia adecuada. Se suspendió la VNI a las 24 horas. Al $5^{\text {to }}$ día

FIgURA 2. Tomografía computada de tórax. Fractura costal, hemoneumotórax, contusión pulmonar bilateral

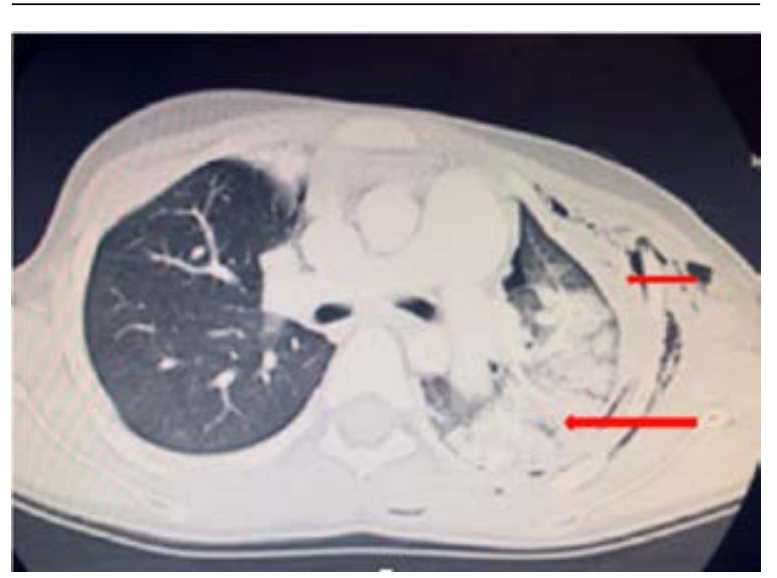


de internación, se retiró el drenaje pleural. La paciente evolucionó favorablemente. Permaneció 5 días en AVM y 9 días de internación en total. En el control posquirúrgico a los 60 días, no se observó deformidad torácica y no presentó limitaciones en la actividad habitual.

\section{DISCUSIÓN}

No existe consenso en cuanto al tratamiento del TI en la edad pediátrica, probablemente, por su baja incidencia. En los adultos, durante las últimas dos décadas, se ha enfocado en el manejo clínico, dirigido a mantener una adecuada ventilación e higiene pulmonar mediante el uso de analgesia y AVM. ${ }^{6,7}$ Esto se ha asociado a la necesidad de uso de analgésicos más potentes, mayor dificultad para la movilización de secreciones y, como consecuencia, la hipoxemia, las infecciones pulmonares graves y atelectasias, entre otras complicaciones. ${ }^{8}$ A largo plazo, se han observado deformidades en la pared torácica, las cuales resultan en insuficiencia pulmonar crónica y dolor pleurítico crónico. ${ }^{1}$

Los últimos avances en el manejo del TI implementan el tratamiento quirúrgico precoz mediante la fijación interna de las fracturas costales. Las indicaciones quirúrgicas incluyen a aquellos pacientes con falla respiratoria a pesar de la analgesia continua adecuada y la limpieza pulmonar, pacientes con deformidad extensa de la pared costal con desplazamiento progresivo de fracturas costales, pacientes con AVM prolongada, inadecuado manejo del dolor

Figura 3 y 4 . Fracturas costales y osteosintesis con placas de Judet

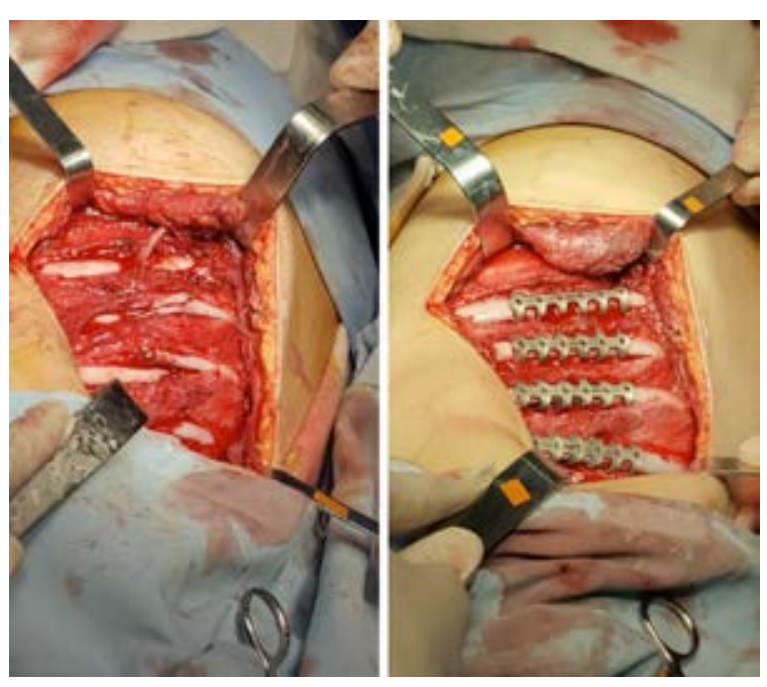

a pesar de la analgesia adecuada y aquellos con lesiones quirúrgicas torácicas asociadas. ${ }^{9}$

Los algoritmos de manejo de TI difieren cuando existe una CP asociada. Esto se debe a estudios que demuestran que la fijación costal no proporciona beneficios a corto plazo cuando el paciente requiere AVM para el tratamiento de una CP clínicamente relevante. ${ }^{10}$ Los casos en los que las CP fueron diagnosticadas por una radiografía de tórax inicial requirieron más apoyo respiratorio (incluida la intubación), estancias más prolongadas en la Unidad de Cuidados Intensivos $\mathrm{y}$ en el hospital que aquellos cuyas $\mathrm{CP}$ solo se diagnosticaron mediante TC. ${ }^{10}$

La presencia de lesiones asociadas, que implican un mayor daño (traumatismo craneoencefálico grave, contusión miocárdica, etc.), aumentan la morbimortalidad del TI, lo que es también un impedimento para la operación quirúrgica. ${ }^{11} \mathrm{El}$ manejo quirúrgico temprano ha demostrado mejores resultados clínicos $\mathrm{y}$, a su vez, la reducción en los costos. ${ }^{13}$ Clínicamente, en los pacientes intervenidos, se observó una disminución en la incidencia de neumonía, en la duración de la AVM, en la estadía en la Unidad de Cuidados Intensivos y en la mortalidad., ${ }^{9} 13$

Estudios realizados ponen evidencia que, 72 horas después de la operación quirúrgica, el dolor causado por la fricción entre los extremos de las fracturas costales se reduce, el patrón respiratorio cambia de superficial a normal y las alteraciones hemodinámicas mejoran significativamente. ${ }^{8}$ En el caso expuesto, la paciente presentaba un TI con CP sin impacto gasométrico y sin compromiso de otro sistema, con fracturas que involucraban desde la $2^{\mathrm{a}}$ a la $7^{a}$ costilla, que tenían un rol central en la estabilidad de la caja torácica, por lo que la fijación quirúrgica precoz fue la elección de tratamiento.

Se han descrito múltiples técnicas quirúrgicas y materiales de osteosíntesis, que incluyen el uso de placas de metal, placas absorbibles, fijadores intramedulares, placas de Judet y placas en U. El tipo y el motivo de la elección del material por utilizar es muy variable y sin consenso al respecto. ${ }^{14,15}$ Se eligió utilizar placas de Judet, debido a que, entre los materiales disponibles, su forma, tamaño y plasticidad para moldearlas permitieron realizar el procedimiento en un paciente pediátrico de manera simple y con mínima invasión para preservar los músculos intercostales, al igual que lo descrito en la bibliografia. ${ }^{15}$ Entre las complicaciones, se 
encuentran la infección de la herida, osteomielitis, falla o migración de la prótesis, mal unión o falta de unión ósea. Ninguna de ellas se presentó en nuestro paciente.

La decisión de implementar AVM en nuestro paciente fue electiva, para el manejo del dolor y para brindar estabilidad a la pared torácica. No existen protocolos de AVM en pacientes pediátricos con $\mathrm{TI}$, por lo que el manejo de los parámetros ventilatorios se realizó a criterio del equipo tratante. Se considera que la estrategia de la resolución quirúrgica temprana permitió un mejor manejo del dolor, lo que posibilitó la rápida suspensión de la $\mathrm{AVM}$ y la reducción de la estadía hospitalaria sin presentar complicaciones en nuestro paciente.

\section{REFERENCIAS}

1. Schuurmans J, Goslings JC, Schepers T. Operative management versus non-operative management of rib fractures in flail chest injuries: a systematic review. Eur $J$ Trauma Emerg Surg. 2017; 43(2):163-8.

2. Xu JQ, Qiu PL, Yu RG, Gong SR, et al. Better short-term efficacy of treating severe flail chest with internal fixation surgery compared with conservative treatments. Eur JMed Res. 2015; $20: 55$.

3. Yasuda R, Okada H, Shirai K, Yoshida S, et al. Comparison of two pediatric flail chest cases. Scand J Trauma Resusc Emerg Med. 2015; 23:73.

4. Vana PG, Neubauer DC, Luchette FA. Contemporary management of flail chest. Am Surg. 2014; 80(6):527-35.

5. Kasotakis G, Hasenboehler EA, Streib EW, Patel N, et al. Operative fixation of rib fractures after blunt trauma: a practice management guideline from the eastern Association for the Surgery of trauma. J Trauma Acute Care Surg. 2017; 82(3):618-26.
6. Doben AR, Eriksson EA, Denlinger CE, Leon SM, et al. Surgical rib fixation for flail chest deformity improves liberation from mechanical ventilation. J Crit Care. 2014; 29(1):139-43.

7. Simon B, Ebert J, Bokhari F, Capella J, et al. Management of pulmonary contusion and flail chest: an Eastern Association for the Surgery of Trauma practice management guideline. J Trauma Acute Care Surg. 2012; 73(5 Suppl 4):S351-61.

8. Liu X, Xiong K. Surgical management versus non-surgical management of rib fractures in chest trauma: a systematic review and meta-analysis.JCardiothorac Surg. 2019;14(1):45.

9. Gerakopoulos E, Walker L, Melling D, ScottS, et al.Surgical management of multiple rib fractures reduces the hospital length of stay and the mortality rate in major trauma patients: a comparative study in a UKMajor Trauma Center. J Orthop Trauma. 2019; 33(1):9-14.

10. Pauzé DR, Pauzé DK. Emergency management of blunt chest trauma in children: an evidence-based approach. Pediatr Emerg Med Pract. 2013; 10:1-22.

11. Battle CE, Evans PA. Predictors of mortality in patients with flail chest: A systematic review. Emerg Med J. 2015; 32(12):961-5.

12. Swart E, Laratta J, Slobogean G, Mehta S. Operative treatment of rib fractures in flail chest injuries: a metaanalysis and cost-effectiveness analysis. J Orthop Trauma. 2017; 31(2):64-70.

13. Coughlin T, Ng JW, Rollins KE, Forward DP, et al. Management of rib fractures in traumatic flail chest: a meta-analysis of randomised controlled trials. Bone Joint J. 2016; 98-B(8):1119-25.

14. Tanaka H, Yukioka T, Yamaguti Y, Shimizu S, et al. Surgical stabilization of internal pneumatic stabilization? A prospective randomized study of management of severe flail chest patients. $J$ Trauma. 2002; 52(4):727-32.

15. Vyhnánek F, Jirava D, Ocadlik M, Skrabalová D, et al. Inovovanéžebernídlahy Judetova typu-preklinickástudie, první klinické zkušenosti. Acta Chir Orthop Traumatol Cech. 2018; 85(3):226-30 